\title{
Consensus Control for a Multiagent System with Time Delays
}

\author{
Yiran Cao, ${ }^{1}$ Toshiki Oguchi, ${ }^{1}$ Paul Verhoeckx, ${ }^{2}$ and Henk Nijmeijer ${ }^{2}$ \\ ${ }^{1}$ Department of Mechanical Engineering, Graduate School of Science and Engineering, Tokyo Metropolitan University, \\ 1-1 Minami-Osawa, Hachioji-shi, Tokyo 192-0397, Japan \\ ${ }^{2}$ Department of Mechanical Engineering, Eindhoven University of Technology, P.O. Box 513, 5600MB Eindhoven, Netherlands \\ Correspondence should be addressed to Yiran Cao; cao-yiran@ed.tmu.ac.jp
}

Received 2 September 2016; Revised 14 December 2016; Accepted 26 December 2016; Published 28 February 2017

Academic Editor: Olfa Boubaker

Copyright (C) 2017 Yiran Cao et al. This is an open access article distributed under the Creative Commons Attribution License, which permits unrestricted use, distribution, and reproduction in any medium, provided the original work is properly cited.

In this paper, we consider the consensus control problem for a multiagent system (MAS) consisting of integrator dynamics with input and output time delays. First, we investigate a consensus condition for the MAS with a linear controller and without any delay compensation. We then propose a consensus controller with a state predictor to compensate the effect of time delay. The consensus condition for this controller is derived and investigated. Finally, we present an example of solving the consensus control problem for two-wheel mobile robots with feedback loops that pass through a computer network with time delays. To demonstrate the validity of the predictor-based controller, we conduct experiments with two-wheel mobile robots and present the results.

\section{Introduction}

Achieving cooperative control of robotic systems is of increasing interest and has attracted a great attention in recent years. There are many potential applications for multirobot systems, including unmanned aerial vehicles, satellite clusters, automated highways, and search and rescue operations. Control tasks for robotic systems include consensus $[1,2]$, flocking [3-5], formation control [6, 7], and tracking [810]. Of these, consensus constitutes a fundamental problem for the coordination control of distributed systems. Since cooperative multirobot systems rely on communication between robots in order to collaborate, time delays due to communication through networks and computations are a problem that cannot be neglected. Time delays in general can degrade system performance or even destroy stability. When each robot is considered to be an individual agent, multirobot systems can be considered multiagent systems (MASs). Here, we consider the consensus problem of MASs with time delay.

For nonlinear systems with input delay, Oguchi and Nijmeijer [11] proposed a delay compensation method with a state predictor based on anticipating synchronization. Several studies $[1,12-16]$ have focused on the consensus problem in a MAS with time delay. The papers $[12,13]$ showed the upper bound of allowable input time delay under which consensus could be achieved. We attempt to consider both input and output time delay due to both communication and computation. In this study, we first introduce a linear time-delay control protocol with a corresponding consensus condition, similar to that in [12]. The consensus condition is used to give the consensus region of the allowable time delay corresponding to coupling strength. To compensate the timedelay effect, we focus on using a prediction control scheme for MASs with input and output time delays to allow the system to achieve consensus. A previously proposed state predictorbased controller for nonlinear systems with time delay is based on anticipating synchronization [11]. Kojima et al. used it for a tracking-control problem with time delay [17], as did Alvarez-Aguirre et al. [18]. In our previous work [2], we used a controller based on this predictor [11] to solve the consensus problem of MASs in undirected graph networks with time delay and derived the corresponding consensus condition. In this paper, by extending the results, we show that the system achieves the average consensus, and the MASs with directed communication graph can also achieve consensus under the consensus condition. To show the validity of the obtained results, we make the experiments in a multirobot system with the proposed controllers to solve the consensus problem of the coordinates of mobile robots. 
This article is organized as follows. In Section 2, we introduce consensus problems for networks of dynamic agents with input and output time delays and show the necessary and sufficient conditions with linear coupling without the predictor to achieve consensus. We then propose a predictor-based consensus controller, for which we derive the necessary and sufficient conditions. Simulation results are presented in Section 3. Section 4 shows experimental results of nonholonomic mobile robots with input and output time delays to show the effectiveness of the proposed control scheme. Finally, Section 5 contains our conclusions.

\section{Problem Formulation}

Consider a network that consists of $N$ identical integrator agents with invariant input and output time delays given as the following dynamics:

$$
\begin{aligned}
& \dot{\mathbf{x}}_{i}(t)=\mathbf{u}_{i}\left(t-\tau_{i}^{\text {in }}\right) \\
& \mathbf{y}_{i}(t)=\mathbf{x}_{i}\left(t-\tau_{i}^{\text {out }}\right)
\end{aligned}
$$

$$
\text { for } i=1,2, \ldots, N \text {. }
$$

Here $\mathbf{x}_{i}=\left[x_{1 i}, x_{2 i}, \ldots, x_{n i}\right]^{T} \in \mathbb{R}^{n}, \mathbf{y}_{i}=\left[y_{1 i}, y_{2 i}, \ldots, y_{n i}\right]^{T} \in$ $\mathbb{R}^{n}$, and $\mathbf{u}_{i}=\left[u_{1 i}, u_{2 i}, \ldots, u_{n i}\right]^{T} \in \mathbb{R}^{n}$ denote the state, output, and input vectors of the $i$ th agent, respectively. $\tau_{i}^{\text {in }}$ and $\tau_{i}^{\text {out }} \in \mathbb{R}^{+}$separately denote the input and output delays corresponding to agent $i$.

For this system, the consensus problem is formulated as follows.

Definition 1 (consensus problem). For multiagent system (1) with input and output time delays, the consensus problem is to find a control protocol to make the states of all agents reach agreement such that $\mathbf{x}_{i}(t)=\mathbf{x}_{j}(t)$ for all $i, j \in\{1, \ldots, N\}$ as $t \rightarrow \infty$.

Following the consensus control protocol proposed by [12], we assume that these agents are interconnected by the following controller:

$$
\mathbf{u}_{i}(t)=-\sum_{j \in \mathscr{N}_{i}} k_{i j}\left(\mathbf{y}_{i}(t)-\mathbf{y}_{j}(t)\right)
$$

for $i=1, \ldots, N$, where $k_{i j} \in \mathbb{R}_{+}$denotes the coupling strength between agents $i$ and $j . \mathcal{N}_{i}$ denotes the set of agents adjacent to agent $i$, which means these agents are connected to agent $i$ in the network topology. We now introduce some definitions about a graph $\mathscr{G} . L(\mathscr{G})$ is the Laplacian matrix of a graph $\mathscr{G}$ corresponding to the network topology constructed by the interconnection of the agents. If the information communication between agent $i$ and $j$ is bidirectional, the graph $\mathscr{G}$ is undirected, and the corresponding Laplacian $L(\mathscr{G})$ has the following entries:

$$
\ell_{i j}= \begin{cases}-1 & \text { if } j \in \mathcal{N}_{i} \\ 0 & \text { if } j \notin \mathscr{N}_{i}, \quad j \neq i \\ \left|\mathcal{N}_{i}\right| & \text { if } j=i .\end{cases}
$$

It is well known that the bidirectional graph Laplacian $L(\mathscr{G})$ is diagonalized and has a zero eigenvalue, and $N-1$ positive real eigenvalues such as $0=\lambda_{0}<\lambda_{1} \leq \cdots \leq \lambda_{N-1}$ corresponding to the $N$ agents system.

Assuming that $\tau_{i}^{\text {in }}=\tau_{i}^{\text {out }}=\tau$, each round-trip time delay is given by $2 \tau$ for $i=1, \ldots, N$. All coupling strengths are identical and denoted as $k$. Controller (2) is simplified as

$$
\mathbf{u}(t)=-k\left(L(\mathscr{G}) \otimes \mathbf{I}_{n}\right) \mathbf{y}(t)
$$

where $\otimes$ denotes the Kronecker product of two matrices and $\mathbf{y}=\left[\mathbf{y}_{1}^{T}, \mathbf{y}_{2}^{T}, \ldots, \mathbf{y}_{N}^{T}\right]^{T} \in \mathbb{R}^{n N}$ and $\mathbf{u}=\left[\mathbf{u}_{1}^{T}, \mathbf{u}_{2}^{T}, \ldots, \mathbf{u}_{N}^{T}\right]^{T} \in$ $\mathbb{R}^{n N}$ denote the output vector and the input vector, respectively.

The dynamics of the total system can then be derived as

$$
\dot{\mathbf{x}}(t)=-k\left(L(\mathscr{G}) \otimes \mathbf{I}_{n}\right) \mathbf{x}(t-2 \tau),
$$

where $\mathbf{x}=\left[\mathbf{x}_{1}^{T}, \ldots, \mathbf{x}_{N}^{T}\right]^{T} \in \mathbb{R}^{n N}$ denotes the state vector. The initial condition of the states is given as $\mathbf{x}(\theta)=\varphi(\theta)(-2 \tau \leq$ $\theta \leq 0)$, where $\varphi(\theta) \in C\left([-2 \tau, 0], \mathbb{R}^{n N}\right)$.

Therefore, from the stability of system (5), the following consensus condition is proven following the results of OlfatiSaber and Murray [12].

Theorem 2. Assume that each system (1) is interconnected by (2) with a coupling strength $k$ and constant input and output time delay $\tau$. The constructed network topology is fixed, undirected, and connected. If the pair $(k, \tau)$ satisfies

$$
0<k \tau<\frac{\pi}{4 \lambda_{N-1}}
$$

the delayed system achieves consensus. Here $\lambda_{N-1}$ is the maximum eigenvalue of $L(\mathscr{G})$ for an $N$-agents system.

Rewriting $k \lambda_{N-1}$ as $\bar{k}$, pairs of $(\bar{k}, \tau)$ satisfying $0<\tau<$ $\pi / 4 \bar{k}$ can stabilise the delayed system. Thus, $\tau$ has a maximum value boundary corresponding to each value of $\bar{k}$. In general, if the number of agents increases, $\lambda_{N-1}$ corresponding to the network structure also tends to increase. Based on $k=$ $\bar{k} / \lambda_{N-1}$, for a fixed time delay $\tau, k$ decreases as $\lambda_{N-1}$ increases, and this slows the convergence rate. Therefore, this condition means that the convergence rate gets slower as the number of agents and the allowable delay increases. To overcome this problem, in the next section, we propose a state predictorbased controller that can counteract the effect of $\lambda_{N-1}$.

Moreover, if the graph is undirected and connected, [12] shows that MAS without time delays achieve the consensus, and the consensus solution is given as the average of the states of all agents; that is, Ave $(x(t))=(1 / N) \sum_{i=1}^{N} \mathbf{x}_{i}(t)$. In [19], the necessary and sufficient condition for an average consensus problem for MAS with nonuniform and asymmetric time delay is given. From these results, we know that the MAS 
in Theorem 2 achieves the average consensus and that the consensus value is given as

$$
\begin{aligned}
\alpha & :=\frac{\mathbf{1}_{N}^{T} \otimes \mathbf{I}_{n}}{N}\left\{\varphi(0)+\int_{-2 \tau}^{0}\left(L(\mathscr{G}) \otimes \mathbf{I}_{n}\right) \varphi(s) d s\right\} \\
& =\frac{\mathbf{1}_{N}^{T} \otimes \mathbf{I}_{n}}{N} \varphi(0),
\end{aligned}
$$

where $\mathbf{1}_{N}=[1, \ldots, 1]^{T} \in \mathbb{R}^{N}$.

\section{Main Results}

Based on the MAS (1) with controller (2), we propose a controller with a state predictor based on anticipating synchronization for the consensus control of agents. We present the consensus controller and discuss its stability problem in this section.

3.1. State Predictor-Based Controller. Anticipating synchronization is a kind of master-slave synchronization. The predictor is constituted by the given system dynamics and coupling of the difference of the system output and delayed predictor states. The dynamics of this predictor can be stated as follows:

$$
\dot{\hat{\mathbf{y}}}(t)=\mathbf{u}(t)-k_{p}(\widehat{\mathbf{y}}(t-2 \tau)-\mathbf{y}(t)),
$$

where $k_{p} \in \mathbb{R}^{+}$is the prediction gain. Meanwhile, $\widehat{\mathbf{y}}=\left[\widehat{\mathbf{y}}_{1}^{T}\right.$, $\left.\ldots, \widehat{\mathbf{y}}_{N}^{T}\right]^{T} \in \mathbb{R}^{n N}$, where $\widehat{\mathbf{y}}_{i}(t)=\left[\widehat{y}_{1 i}(t), \ldots, \widehat{y}_{n i}(t)\right]^{T} \in \mathbb{R}^{n}, i \in$ $\{1, \ldots, N\}$ denotes the predicted outputs. The initial condition of the predicted states is given as $\widehat{\mathbf{y}}(\theta)=\phi(\theta)(-2 \tau \leq \theta \leq$ $0)$, where $\phi(\theta) \in C\left([-2 \tau, 0], \mathbb{R}^{n N}\right)$. Then, using the output of the predictor instead of the output of the actual system, the main controller is given as

$$
\mathbf{u}(t)=-k\left(L(\mathscr{G}) \otimes \mathbf{I}_{n}\right) \widehat{\mathbf{y}}(t) .
$$

Controller (9) with state predictor (8) compensates the effect of time delays at input and output. If the predictor has prior knowledge of the initial states of the system, the prediction error always remains 0 , and the predictor can predict the exact future value of the states of the system. Thereafter, the total system is shown as

$$
\begin{aligned}
\dot{\mathbf{x}}(t) & =\mathbf{u}(t-\tau), \\
\mathbf{y}(t) & =\mathbf{x}(t-\tau), \\
\mathbf{u}(t) & =-k\left(L(\mathscr{G}) \otimes \mathbf{I}_{n}\right) \widehat{\mathbf{y}}(t), \\
\dot{\hat{\mathbf{y}}}(t) & =\mathbf{u}(t)-k_{p}(\widehat{\mathbf{y}}(t-2 \tau)-\mathbf{y}(t)) .
\end{aligned}
$$

3.2. Consensus Condition. As we use a predictor to predict the states, it is important to prove that the prediction error converges to 0 . The prediction error is defined as

$$
\widehat{\mathbf{e}}(t)=\widehat{\mathbf{y}}(t-\tau)-\mathbf{x}(t) .
$$

When the prediction error $\widehat{\mathbf{y}}(t-\tau)-\mathbf{x}(t)$ converges to zero, this means that $\widehat{\mathbf{y}}(t)$ estimates the exact future value of $\mathbf{x}(t)$, which is $\mathbf{x}(t+\tau)$. The time-delay $2 \tau$ is totally compensated at this time.

With the use of (8) and (9), the dynamics of prediction error can be obtained as

$$
\dot{\hat{\mathbf{e}}}(t)=-k_{p} \widehat{\mathbf{e}}(t-2 \tau) \text {. }
$$

To derive the necessary and sufficient conditions such that the whole system converges to consensus, we consider the coordinate transformation as follows:

$$
\begin{aligned}
\mathbf{e}_{s}(t) & =\left[\begin{array}{c}
\sum_{i=1}^{N} \mathbf{x}_{i}(t) \\
\mathbf{e}(t)
\end{array}\right]=\left[\begin{array}{c}
\mathbf{x}_{1}(t)+\cdots+\mathbf{x}_{N}(t) \\
\mathbf{x}_{1}(t)-\mathbf{x}_{2}(t) \\
\vdots \\
\mathbf{x}_{1}(t)-\mathbf{x}_{N}(t)
\end{array}\right] \\
& =\left(\mathbf{M}_{0} \otimes \mathbf{I}_{n}\right) \mathbf{x}(t),
\end{aligned}
$$

where

$$
\mathbf{M}_{0}=\left[\begin{array}{cccc}
1 & 1 & \cdots & 1 \\
1 & -1 & & 0 \\
\vdots & & \ddots & \\
1 & 0 & & -1
\end{array}\right] \in \mathbb{R}^{N \times N}
$$

and $\mathbf{e}(t)=\left[\mathbf{x}_{1}(t)-\mathbf{x}_{2}(t), \ldots, \mathbf{x}_{1}(t)-\mathbf{x}_{N}(t)\right]^{T}$ denotes the synchronization error. Substituting (8), (9), and (11) for the derivative of (13), we obtain the following dynamics:

$$
\begin{aligned}
\dot{\mathbf{e}}_{s}(t)= & -k\left(\mathbf{M}_{0} L(\mathscr{G}) \mathbf{M}_{0}^{-1} \otimes \mathbf{I}_{n}\right) \mathbf{e}_{s}(t) \\
& -k\left(\mathbf{M}_{0} L(\mathscr{G}) \otimes \mathbf{I}_{n}\right) \widehat{\mathbf{e}}(t) .
\end{aligned}
$$

The prediction error dynamics (12) and the dynamics (15) can be rewritten in a matrix form as

$$
\begin{aligned}
& {\left[\begin{array}{c}
\dot{\mathbf{e}}_{s}(t) \\
\dot{\hat{\mathbf{e}}}(t)
\end{array}\right]=\left[\begin{array}{cc}
0 & 0 \\
0 & -k_{p}
\end{array}\right]\left[\begin{array}{c}
\mathbf{e}_{s}(t-2 \tau) \\
\hat{\mathbf{e}}(t-2 \tau)
\end{array}\right]} \\
& +\left[\begin{array}{cc}
-k\left(\mathbf{M}_{0} L(\mathscr{G}) \mathbf{M}_{0}^{-1} \otimes \mathbf{I}_{n}\right) & -k\left(\mathbf{M}_{0} L(\mathscr{G}) \otimes \mathbf{I}_{n}\right) \\
0 & 0
\end{array}\right]\left[\begin{array}{c}
\mathbf{e}_{s}(t) \\
\widehat{\mathbf{e}}(t)
\end{array}\right] .
\end{aligned}
$$

From this equation, the consensus condition is given in the following theorem.

Theorem 3. Assume that each agent (1) is controlled by predictor (8) and controller (9) with gain $k$, constant input and output time delay $\tau$ and prediction gain $k_{p}$. The constructed network topology is fixed, undirected, and connected. Then, if the pair $\left(k_{p}, \tau\right)$ and $k$ satisfy

$$
\begin{aligned}
& k>0, \\
& 0<k_{p} \tau<\frac{\pi}{4},
\end{aligned}
$$

the MAS achieves consensus. 
Proof. The proof is given for the stability of the total synchronization error dynamics (16). After the Laplace transformation, the characteristic equation of (16) can be derived as

$$
\begin{aligned}
& \operatorname{det}\left[\begin{array}{cc}
s \mathbf{I}_{n N}+k\left(\mathbf{M}_{0} L(\mathscr{G}) \mathbf{M}_{0}^{-1} \otimes \mathbf{I}_{n}\right) & k\left(\mathbf{M}_{0} L(\mathscr{G}) \otimes \mathbf{I}_{n}\right) \\
0 & s \mathbf{I}_{n N}+k_{p}\left(\mathbf{e}^{-2 \tau s} \mathbf{I}_{n N}\right)
\end{array}\right] \\
& =\operatorname{det}\left(s \mathbf{I}_{n N}+k\left(\mathbf{M}_{0} L(\mathscr{G}) \mathbf{M}_{0}^{-1}\right) \otimes \mathbf{I}_{n}\right) \\
& \cdot \operatorname{det}\left(s \mathbf{I}_{n N}+k_{p}\left(\mathbf{e}^{-2 \tau s} \mathbf{I}_{n N}\right)\right)=0 .
\end{aligned}
$$

To make the equation hold, one of the above determinates should be equal to 0 . As can be seen, the first determinant of (18) represents the synchronization error, and the latter represents the prediction error. To make both errors converge to 0 , all solutions satisfying the following equations must have negative real parts:

$$
\begin{array}{r}
\operatorname{det}\left(s \mathbf{I}_{n N}+k\left(\mathbf{M}_{0} L(\mathscr{G}) \mathbf{M}_{0}^{-1}\right) \otimes \mathbf{I}_{n}\right)=0, \\
\operatorname{det}\left(s \mathbf{I}_{n N}+k_{p}\left(\mathbf{e}^{-2 \tau s} \mathbf{I}_{n N}\right)\right)=0 .
\end{array}
$$

Since $L(\mathscr{G})$ for a fixed, undirected, and connected graph is a symmetric real matrix and for the eigenvalues it holds that $0<\lambda_{1} \leq \cdots \leq \lambda_{N-1}$, the term of the first equation in (19) satisfies the following results:

$$
\mathbf{P M}_{0} L(\mathscr{G}) \mathbf{M}_{0}^{-1} \mathbf{P}^{-1}=\left[\begin{array}{c|ccc}
0 & 0 & \cdots & 0 \\
\hline 0 & \lambda_{1} & \cdots & 0 \\
\vdots & \vdots & \ddots & \vdots \\
0 & 0 & \cdots & \lambda_{N-1}
\end{array}\right]
$$

where $\mathbf{P} \in \mathbb{R}^{N \times N}$ is the matrix that transforms $\mathbf{M}_{0} L(\mathscr{G}) \mathbf{M}_{0}^{-1}$ into the diagonalized form. Thus, the first equation can be written as $s+k \lambda_{i}=0, i=1, \ldots, N-1$. To make $s$ have negative real part, we get the condition $k>0$ from the first equation.

For the second equation, we consider the smallest value of $\tau$, such that $s=j \omega$, which has a zero real part on the imaginary axis. Then we have

$$
\begin{aligned}
\omega-k_{p} \sin (\omega \tau) & =0, \\
k_{p} \cos (\omega \tau) & =0 .
\end{aligned}
$$

Assuming $\omega>0$, we can get $\omega \tau=(\pi / 2)+2 k \pi, k=$ $0,1, \ldots, N$ and $\omega=k_{p}$. Since the delay-free system is described by $s+k_{p}=0$ (which is exponentially stable for $k_{p}>0$ ), and the continuity of eigenvalues for LTI systems holds, the roots of the dynamics of the second equation of (19) lie on the open left half-plane. Therefore, from the second equation, we get $0<k_{p} \tau<\pi / 2$.

The first condition corresponds to the consensus condition for the system without delay, and the second comes from the stability of the prediction error. This discussion means that the synchronization-based predictor is an extension of the full-state observer, and a counterpart of the separation principle holds for the stability of the system with the synchronization-based predictor.

Compared with the consensus condition (6) of Theorem 2, the ranges of both $k$ and $\tau$ are extended. The coupling strength $k$ is independent of time delay in Theorem 3 and $k_{p}<\pi / 4 \tau$ holds for any constant $\tau$. Moreover, if the prediction error is not zero, both $k$ and $k_{p}$ affect the convergence rate. If the predictor has prior knowledge of the initial condition, $k$ is the only influence factor for the convergence rate. We can choose a larger value of $k$ in order to make the system converge to consensus faster.

Remark 4. It is known that a directed graph contains a directed spanning tree, if and only if the corresponding graph Laplacian $L(\mathscr{G})$ always has one zero eigenvalue and $N-1$ eigenvalues that have positive real parts [20]. In this network topology, the MAS satisfying inequalities (17) can also reach a consensus.

Concerning the average value of agent states, we have the following results.

Theorem 5. Consider that the system with agent (1) satisfies Theorem 3, so that the MAS achieves consensus. This MAS achieves average consensus for any initial states $\varphi(0) \in \mathbb{R}^{n N}$ and the consensus solution is given as

$$
\boldsymbol{\alpha}_{p}:=\frac{\mathbf{1}_{N}^{T} \otimes \mathbf{I}_{n}}{N} \varphi(0) .
$$

Proof. The total system can be summarised as

$$
\begin{aligned}
\dot{\mathbf{x}}(t)= & -k\left(L(\mathscr{G}) \otimes \mathbf{I}_{n}\right) \widehat{\mathbf{y}}(t-\tau), \\
\dot{\hat{\mathbf{y}}}(t)= & -k\left(L(\mathscr{G}) \otimes \mathbf{I}_{n}\right) \widehat{\mathbf{y}}(t) \\
& -k_{p}(\widehat{\mathbf{y}}(t-2 \tau)-\mathbf{x}(t-\tau)) .
\end{aligned}
$$

Following the method shown in [19], we consider the following functional vector $F_{1}: C\left([-\tau, 0], \mathbb{R}^{n N}\right) \rightarrow \mathbb{R}^{n}$ :

$$
\begin{aligned}
& F_{1}\left(\mathbf{x}_{t}\right)=\frac{1}{N}\left(\mathbf{1}_{\mathrm{N}}^{T} \otimes \mathbf{I}_{n}\right) \\
& \cdot\left(\mathbf{x}(t)+\int_{t-\tau}^{t}-k\left(L(\mathscr{G}) \otimes \mathbf{I}_{n}\right) \widehat{\mathbf{y}}(s) d s\right) \\
& =\frac{1}{N}\left(\mathbf{1}_{N}^{T} \otimes \mathbf{I}_{n}\right) \mathbf{x}(t)
\end{aligned}
$$

where $\mathbf{x}_{t} \in C\left([-\tau, 0], \mathbb{R}^{n N}\right)$ represents the solution $\mathbf{x}(t) \epsilon$ $\mathbb{R}^{n N}$ on the time interval $[t-\tau, t]$ such that $\mathbf{x}_{t}(s)=\mathbf{x}(t+s), s \in$ $[-\tau, 0]$. Since this graph Laplacian $L(\mathscr{G})$ is a symmetric zero column-sum matrix, $\mathbf{1}_{N}^{T} L(\mathscr{G})=\mathbf{0}$ holds. Thus, the integral 


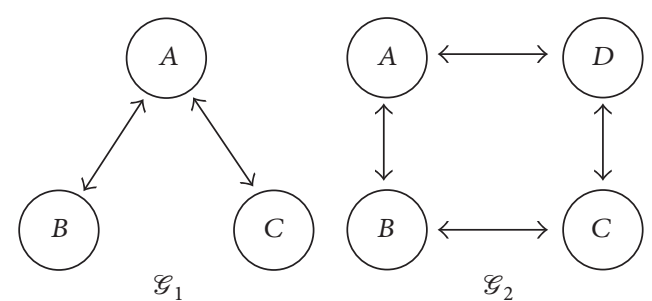

FIGURE 1: Network structures for three and four agents.

term is vanished in (24). The time-derivative of (24) along the solution of (23) is given as

$$
\begin{aligned}
& \frac{d}{d t} F_{1}\left(\mathbf{x}_{t}\right)=\frac{1}{N}\left(\mathbf{1}_{N}^{T} \otimes \mathbf{I}_{n}\right) \\
& \cdot\left(\mathbf{x}(t)+\int_{t-\tau}^{t}-k\left(L(\mathscr{G}) \otimes \mathbf{I}_{n}\right) \widehat{\mathbf{y}}(s) d s\right) \\
& \quad=\frac{1}{N}\left(\mathbf{1}_{N}^{T} \otimes \mathbf{I}_{n}\right)\left(-k\left(L(\mathscr{G}) \otimes \mathbf{I}_{n}\right) \widehat{\mathbf{y}}(t-\tau)\right)=0 .
\end{aligned}
$$

Therefore, the functional vector $F_{1}\left(\mathbf{x}_{t}\right)$ is time-invariant, and the value always equals the average of the initial state $\mathbf{x}(0)$ given as

$$
\begin{aligned}
F_{1}\left(\mathbf{x}_{t}\right) & =\frac{\mathbf{1}_{N}^{T} \otimes \mathbf{I}_{n}}{N} \mathbf{I}_{n N}\left(\mathbf{1}_{N} \otimes \boldsymbol{\alpha}_{p}\right)=\boldsymbol{\alpha}_{p} \\
& =\frac{\mathbf{1}_{N}^{T} \otimes \mathbf{I}_{n}}{N} \boldsymbol{\varphi}(0) .
\end{aligned}
$$

3.3. Simulation Results. By using controller (9) and predictor (8), we can obtain the simulation results for the system (10) of three and four agents, respectively, connected by networks in Figure 1.

The graph Laplacian $L\left(\mathscr{G}_{1}\right)$ for three agents is

$$
L\left(\mathscr{G}_{1}\right)=\left[\begin{array}{ccc}
2 & -1 & -1 \\
-1 & 1 & 0 \\
-1 & 0 & 1
\end{array}\right]
$$

and the corresponding eigenvalues are $\lambda_{i}=[0,1,3], \quad i=$ $0,1,2$.

In this simulation the coupling strength is $k=2$, the prediction gain $k_{p}=0.8$, and the time-delay is set as $\tau=$ $0.25 \mathrm{~s}$. The initial states are set as $\mathbf{x}_{A}=[1.35,2.54]^{T}, \mathbf{x}_{B}=$ $[0.98,2.99]^{T}$, and $\mathbf{x}_{C}=[1.60,3.07]^{T}$ for each agent, and the initial states of the predictor are given as $\widehat{\mathbf{y}}(t)=\mathbf{0}$ for $-2 \tau \leq$ $t \leq 0$. The simulation results are shown in Figure 2. It is clearly shown that both the prediction error and the synchronization error converge to 0 for each state. The consensus values of the system are the average of the states at $t=0$; that is, $\boldsymbol{\alpha}_{p}=[3.93,2.9]^{T}$.
For a four-agent system, the graph Laplacian $L\left(\mathscr{G}_{2}\right)$ of the communication network in Figure 1 is

$$
L\left(\mathscr{G}_{2}\right)=\left[\begin{array}{cccc}
2 & -1 & 0 & -1 \\
-1 & 2 & -1 & 0 \\
0 & -1 & 2 & -1 \\
-1 & 0 & -1 & 2
\end{array}\right]
$$

and the corresponding eigenvalues are $\lambda_{i}=[0,2,2,4], i=$ $0,1,2,3$. The time delay is set as $\tau=1 \mathrm{~s}$, coupling strength $k=2$, and prediction gain $k_{p}=0.4$ satisfying the consensus condition shown in Theorem 3 . We set $\widehat{\mathbf{y}}(t)=\mathbf{0}$ for $-2 \tau \leq$ $t \leq 0$ as the initial states of the predictor; the initial states of dynamics (1) are $\mathbf{x}_{A}=[1.12,2.56]^{T}, \mathbf{x}_{B}=[1.62,2.57]^{T}$, $\mathbf{x}_{C}=[1.59,3.06]^{T}$, and $\mathbf{x}_{D}=[1.15,3.06]^{T}$ for the four agents, respectively.

With more agents and a longer time delay, the MAS satisfies Theorem 3 and converges to consensus, and the prediction error converges to 0 , as shown in Figure 3. Since the simulation results show that the two MASs all converge to consensus under the conditions given in Theorem 3, the validity of the predictor-based controller is verified.

\section{Experimental Results}

In this section, by applying controller (9) with state predictor (8), we show experimental results for multirobot systems.

Consider the two-wheel mobile robot shown in Figure 4. Let us suppose that the robot moves on a flat plane under a fixed global frame without drift. Let $\xi_{i}(t)$ and $\zeta_{i}(t)$ denote the global coordinates of the centre of the $i$ th mobile robot; $\theta_{i}(t)$ denotes the current angle between the direction of velocity $v_{i}(t)$ of the centre and the $x$-axis, and $\omega_{i}(t)=d \theta_{i}(t) / d t$ is the angular velocity. The kinematic model of the $i$ th mobile robot is expressed as

$$
\left[\begin{array}{l}
\dot{\xi}_{i}(t) \\
\dot{\zeta}_{i}(t) \\
\dot{\theta}_{i}(t)
\end{array}\right]=\left[\begin{array}{cc}
\cos \theta_{i}(t) & 0 \\
\sin \theta_{i}(t) & 0 \\
0 & 1
\end{array}\right]\left[\begin{array}{c}
v_{i}(t) \\
\omega_{i}(t)
\end{array}\right]
$$

for $i=1, \ldots, N$. Then, the coordinate $\left(\xi_{o}^{i}(t), \zeta_{o}^{i}(t)\right)$ of point $O^{i}$ on the head of the robot used as the outputs is represented by the coordinate transformation

$$
\left[\begin{array}{l}
\xi_{o}^{i}(t) \\
\zeta_{o}^{i}(t)
\end{array}\right]=\left[\begin{array}{l}
\xi_{i}(t)+R \cos \theta_{i}(t) \\
\zeta_{i}(t)+R \sin \theta_{i}(t)
\end{array}\right],
$$

where $R \in \mathbb{R}^{+}$is the radius of the mobile robot.

Applying (30) into (29), we obtain

$$
\left[\begin{array}{c}
\dot{\xi}_{o}^{i}(t) \\
\dot{\zeta}_{o}^{i}(t)
\end{array}\right]=\left[\begin{array}{cc}
\cos \theta_{i}(t) & -R \sin \theta_{i}(t) \\
\sin \theta_{i}(t) & R \cos \theta_{i}(t)
\end{array}\right]\left[\begin{array}{c}
v_{i}(t) \\
\omega_{i}(t)
\end{array}\right] .
$$




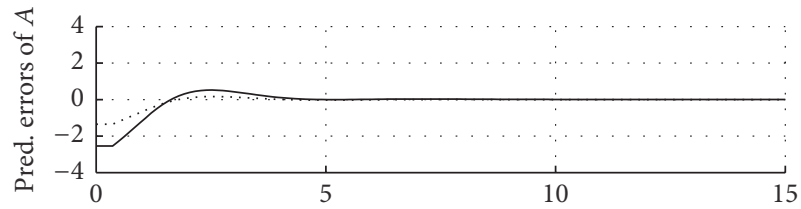

$t(\mathrm{~s})$
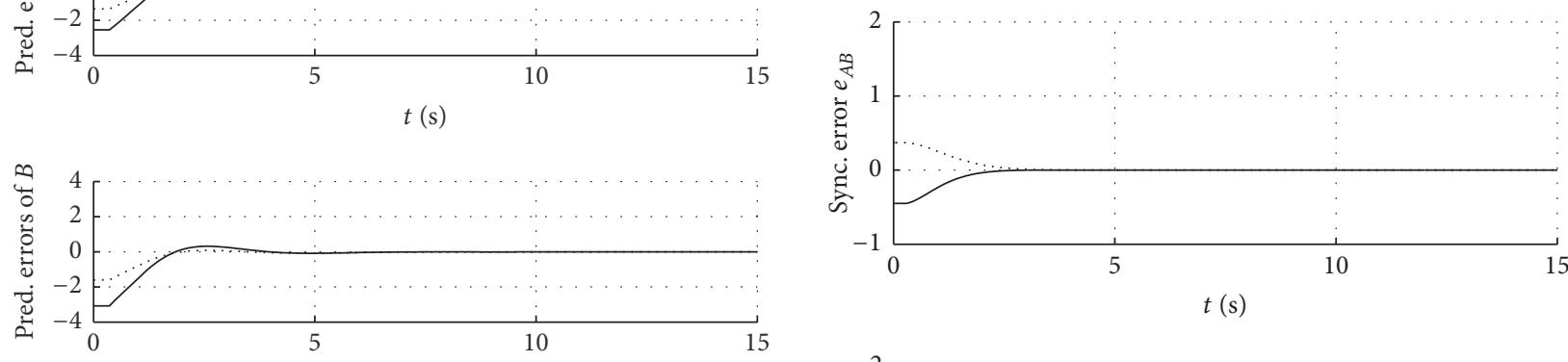

$t(\mathrm{~s})$
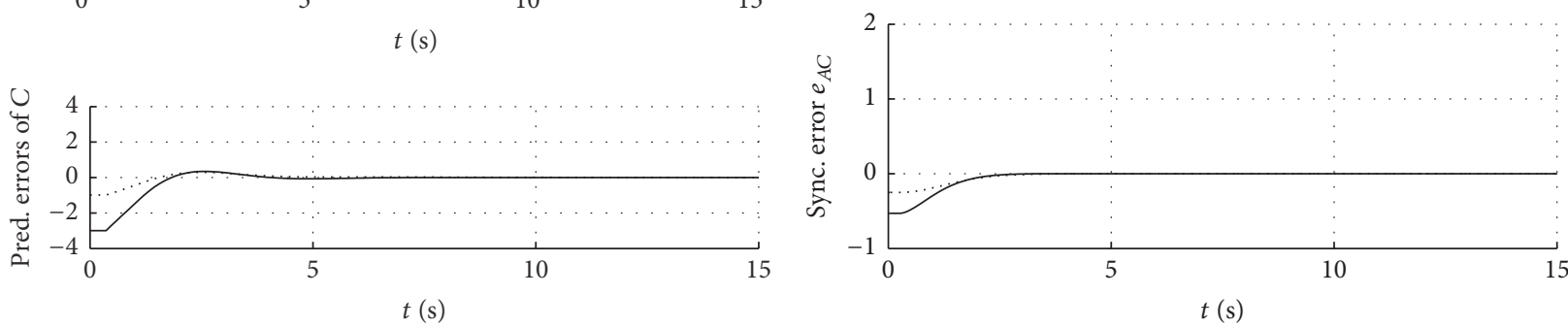

…. $x_{1 i}$

…. $x_{1 i}$

(a) Prediction error

(b) Synchronization error

FIGURE 2: Simulation results for three agents with controller (9) and state predictor (8).
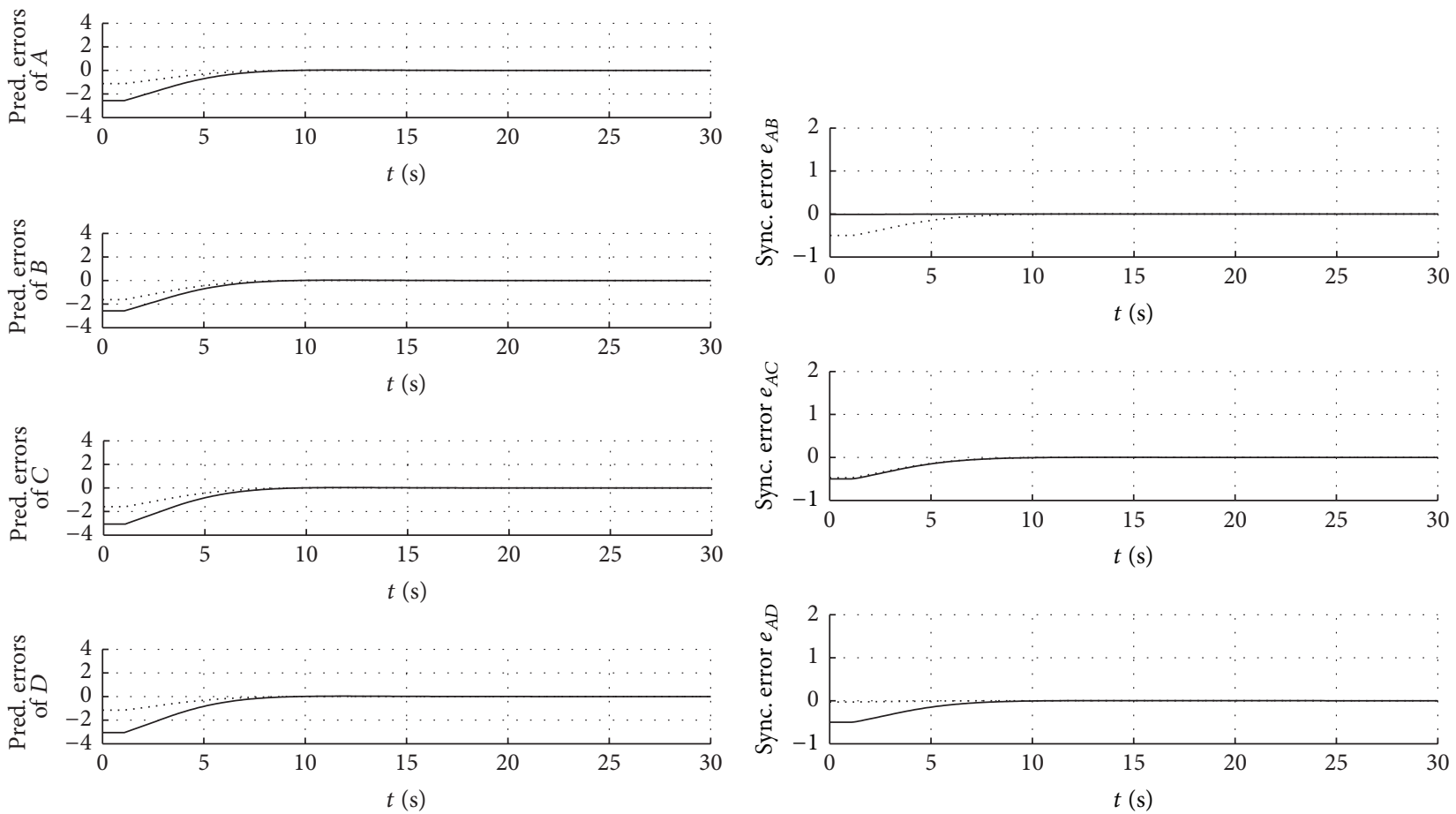

$\begin{array}{rr}\ldots \ldots & x_{1 i} \\ - & x_{2 i}\end{array}$

$\begin{array}{ll}\cdots \cdots & x_{1 i} \\ - & x_{2 i}\end{array}$

(a) Prediction error

(b) Synchronization error

FIGURE 3: Simulation results for four agents with controller (9) and state predictor (8). 


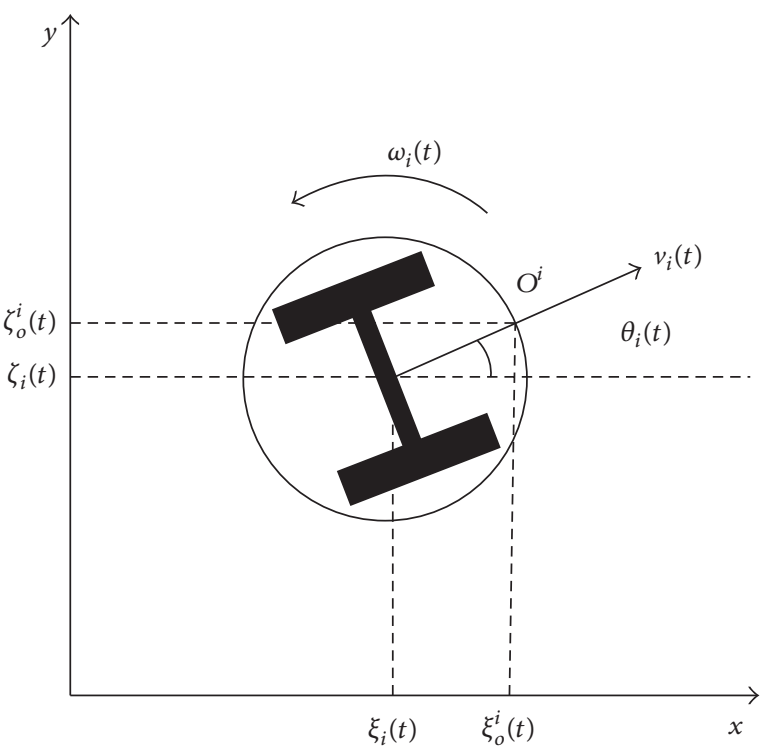

FIGURE 4: Kinematic model of a mobile robot.

For clarity, we simplify $\left[\xi_{o}^{i}(t), \zeta_{o}^{i}(t)\right]^{T}$ as $\mathbf{x}_{i}(t)=\left[\xi_{i}(t)\right.$, $\left.\zeta_{i}(t)\right]^{T}$ for the $i$ th mobile robot. Then (31) can be rewritten as

$$
\begin{aligned}
\dot{\mathbf{x}}_{i}(t) & =\left[\begin{array}{l}
\dot{\zeta}_{i}(t) \\
\dot{\zeta}_{i}(t)
\end{array}\right]=\left[\begin{array}{cc}
\cos \theta_{i}(t) & -R \sin \theta_{i}(t) \\
\sin \theta_{i}(t) & R \cos \theta_{i}(t)
\end{array}\right]\left[\begin{array}{c}
v_{i}(t) \\
\omega_{i}(t)
\end{array}\right] \\
& =\mathbf{B}\left(\theta_{i}(t)\right) \mathbf{p}_{i}(t),
\end{aligned}
$$

where $\mathbf{p}_{i}(t)=\left[v_{i}(t), \omega_{i}(t)\right]^{T}$. Since $\operatorname{det}\left(\mathbf{B}\left(\theta_{i}(t)\right)\right)=R$, the matrix $\mathbf{B}\left(\theta_{i}(t)\right)$ is invertible for any $\theta_{i}(t)$.

The schematic for the system in the experiment is depicted in Figure 5. Time delay occurs in the communication between each robot and a centralized controller. Such a configuration corresponds with a system that has a separate central controller and several local controllers as simple onboard controllers. By using this system, each robot needs only weak computation capability for the local controller, which makes the robots smaller and cheaper. We implement the delayed system by using e-pucks [21], which are two-wheel mobile robots.

We assume that there is a unified constant input and output time delay $\tau$ between each robot and a centralized controller. Applying the input-output feedback linearization technique, we design the local controller carried by each robot as follows:

$$
\mathbf{p}_{i}(t)=\mathbf{B}^{-1}\left(\theta_{i}(t)\right) \mathbf{u}_{i}(t-\tau)
$$

where $\mathbf{u}_{i}(t-\tau) \in \mathbb{R}^{2}$ denotes the output from the centralized controller with time delay $\tau \in \mathbb{R}^{+}$. In this way, $\theta_{i}(t)$ is the local information without time delay for each robot $i$. The differential of an angular $\dot{\theta}_{i}(t)=\omega_{i}(t)$ depends on the control input $\omega_{i}(t) ; \theta_{i}(t)$ is a variant local state, and $\dot{\theta}_{i}(t)$ converges to zero when the control input $\omega_{i}(t)$ converges to zero. By

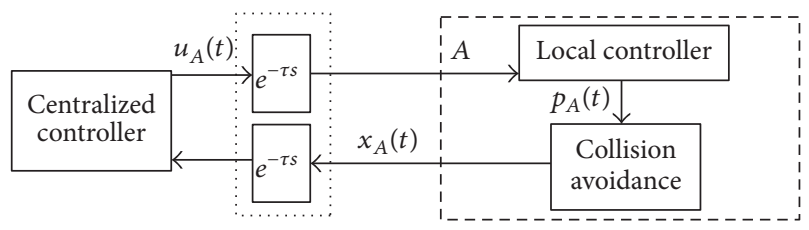

FIGURE 5: Schematic of the robot $A$ system. The local controller and collision avoidance are on-board the robot. Time delay happens on communications between $\operatorname{robot} A$ and the centralized controller.

applying (33) to (32), the system is linearized as an integrator system with input and output time delay given as

$$
\begin{aligned}
\dot{\mathbf{x}}_{i}(t) & =\mathbf{u}_{i}(t-\tau), \\
\mathbf{y}_{i}(t) & =\mathbf{x}_{i}(t-\tau)
\end{aligned}
$$

for $i=1, \ldots, N$. Then the predictor-based control scheme can be used for this multirobot system to converge the output of the robot to consensus.

4.1. Collision Avoidance. On the way to convergence consensus, a robot may collide with other robots. Here, we assume for robot $i$ that the other robots are obstacles. The robot $j$ in danger of colliding with robot $i$ is denoted as belonging to the set

$$
M_{i}=\left\{\mathbf{x}_{j}(t) \mid x_{i j}^{2}(t)<d_{\min }^{2}, j \neq i(j=1, \ldots, N)\right\},
$$

where $x_{i j}^{2}(t)=\left(\xi_{i}(t)-\xi_{j}(t)\right)^{2}+\left(\zeta_{i}(t)-\zeta_{j}(t)\right)^{2}$ denotes the square of the distance between robots $i$ and $j$ and $d_{\min }$ is the minimum safe distance between two robots. Here, the following RPF proposed in [22] is adopted as

$$
V_{i j}(t)= \begin{cases}\eta\left(\frac{1}{x_{i j}^{2}(t)}-\frac{1}{d^{2}}\right)^{2} & \text { if } x_{i j}(t)<d_{\text {min }} \\ 0 & \text { if } x_{i j}(t) \geq d_{\text {min }}\end{cases}
$$

where $\eta \in \mathbb{R}^{+}$is the gain of the RPF. The collision avoidance algorithm can be implemented by the following local controller:

$$
\begin{aligned}
& \mathbf{u}_{i}(t)=-\sum_{j \in M_{i}} \frac{\partial V_{i j}(t)}{\partial \mathbf{x}_{i}(t)} \\
& =4 \sum_{j \in M_{i}} \eta\left(\frac{1}{x_{i j}^{2}(t)}-\frac{1}{d^{2}}\right)\left(\frac{1}{x_{i j}^{2}(t)}\right)^{2}\left(\mathbf{x}_{i}(t)-\mathbf{x}_{j}(t)\right) .
\end{aligned}
$$

Here, the distance $x_{i j}(t)$ is measured by sensing under the measuring range of robot $i$ and the angle $\theta_{i}(t)$ is available as local information. With such local information, the RPF approach can be utilized without time delay, as in $\mathbf{x}_{i}(t)-\mathbf{x}_{j}(t)=\left[x_{i j}(t) \cos \left(\theta_{i}(t)\right), x_{i j}(t) \sin \left(\theta_{i}(t)\right)\right]^{T}$. With 


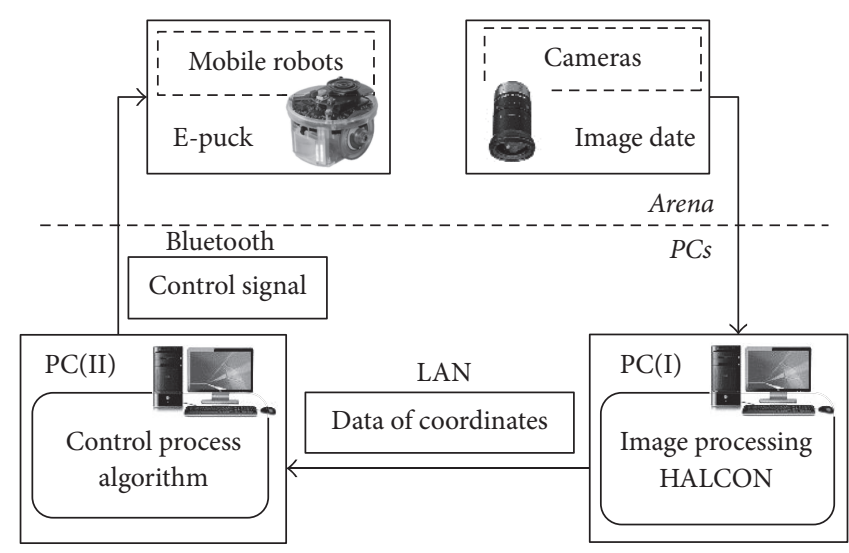

FIGURE 6: Structure of experimental system.

collision avoidance, the controller for the experiment is given

as

$$
\begin{aligned}
& \mathbf{p}_{i}(t)= \mathbf{B}^{-1}\left(\theta_{i}(t)\right) \mathbf{u}_{i}(t-\tau), \\
& \dot{\hat{\mathbf{y}}}_{i}(t)= \mathbf{u}_{i}(t)-k_{p}\left(\widehat{\mathbf{y}}_{i}(t-2 \tau)-\mathbf{y}_{i}(t)\right), \\
& \mathbf{u}_{i}(t)= \begin{cases}-k \sum_{j \in N_{i}}\left(\widehat{\mathbf{y}}_{i}(t)-\widehat{\mathbf{y}}_{j}(t)\right) & \text { if } x_{i j}(t)>d_{\max } \\
0 & \text { if } d_{\min } \leq x_{i j}(t) \leq d_{\max } \\
4 \sum_{j \in M_{i}} \eta\left(\frac{1}{x_{i j}^{2}(t)}-\frac{1}{d^{2}}\right)\left(\frac{1}{x_{i j}^{2}(t)}\right)^{2}\left(\mathbf{x}_{i}(t)-\mathbf{x}_{j}(t)\right) & \text { if } x_{i j}(t)<d_{\min }\end{cases}
\end{aligned}
$$

for $i=1, \ldots, N$.

The consensus problem is to converge the coordinates of point $O^{i}$ on mobile robots to one point. However, considering the possibility of collision, we first use $\Delta r$ as the transformed distance instead of the radius $R$ of the robot. Thus, the transformed coordinates are on the line between point $O^{i}$ and the centre of the robot. Then, if $x_{i j}(t) \in\left[d_{\min }, d_{\max }\right]$ for all $i, j=1, \ldots, N$, the robots are considered to have achieved consensus, and the control program stops. Since the actual volume of the robot should be considered, $d_{\min }=2 R-2 \triangle r+\delta$ and $d_{\max }=2 R+2 \Delta r+\delta$, where $\delta$ is the tolerance.

4.2. Experiment Configuration. As shown in Figure 6, the experimental setup is composed of robots named e-puck, which move on a smooth plane with two overhead CCD cameras to obtain the images of the robots moving. PC(i) is used to analyse the position and angle information of the robots by image processing designed using the software HALCON, and another PC(ii) is applied to calculate the control input $u_{i}(t)$ and send the control signal to each robot through a Bluetooth module.

In the experiment, the radius of each robot is $R=$ $37.5 \mathrm{~mm}$, the transformed distance is set as $\Delta r=5 \mathrm{~mm}$, and the tolerance is set as $\delta=10 \mathrm{~mm}$. Therefore, $d_{\max }$ and $d_{\min }$ in (37) are defined as $d_{\max }=95 \mathrm{~mm}$ and $d_{\min }=75 \mathrm{~mm}$. The gain of RPF is given as $\eta=0.002$. To show the effect of the predictor, time delays are set artificially.

Figure 7 shows the experimental results in the graph topology of the three robots in Figure 1 using controller (40). The initial states, $\left(\xi_{i}(0), \zeta_{i}(0), \boldsymbol{\theta}_{i}(0)\right)$, are $A(1.35,2.54,1.33)$, $B(0.98,2.99,-0.29)$, and $C(1.60,3.07,-2.13)$, respectively. The design parameters are set as the coupling strength $k=2$ and prediction gain $k_{p}=0.8$. Time delay is set as $\tau=0.25 \mathrm{~s}$ artificially. The initial states of the predictor are set as $\widehat{\mathbf{y}}(t)=\mathbf{0}$ for $-2 \tau \leq t \leq 0$.

In Figure $7(\mathrm{a})$, the $\times$ marks indicate the starting positions and the solid circles show the final positions of the robots in which the radius of the small circles is $\Delta r$ and the transformed coordinates are on these circles. Both the final positions of the robots and the synchronization errors given in Figure 7(b) show that the robots converged to consensus intuitively.

Figure 8 shows the experimental results for controller (40) for four e-pucks in the graph topology in Figure 1. The initial coordinates are $A(1.12,2.56,0.98), B(1.62,2.57,2.46)$, $C(1.59,3.06,-2.29)$, and $D(1.15,3.06,-0.84)$ for the four robots, respectively, and we set $\widehat{\mathbf{y}}(t)=\mathbf{0}$ for $-2 \tau \leq t \leq 0$ as the initial state of the predictor. Time delay is set as $\tau=1 \mathrm{~s}$ artificially, coupling strength is $k=2$, and prediction gain $k_{p}=0.4$. 


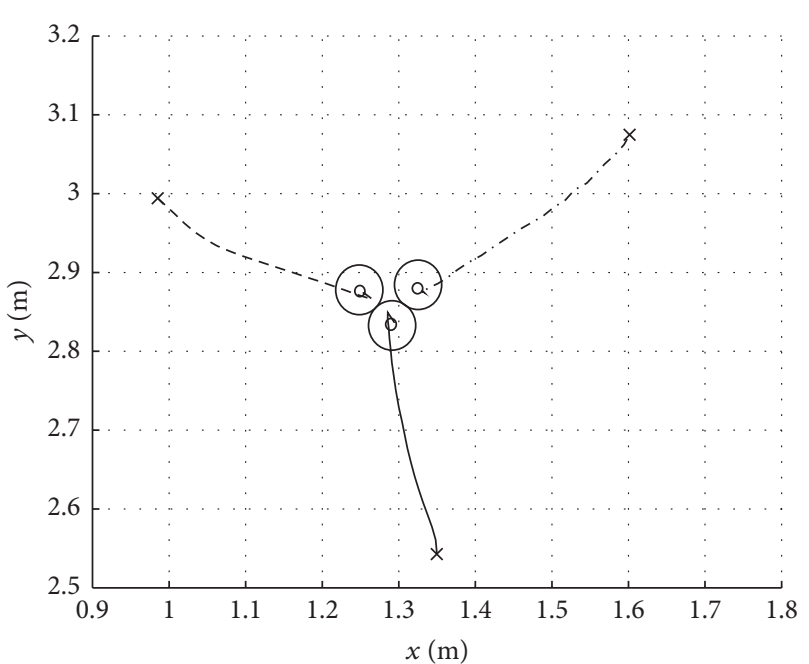

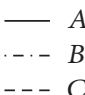
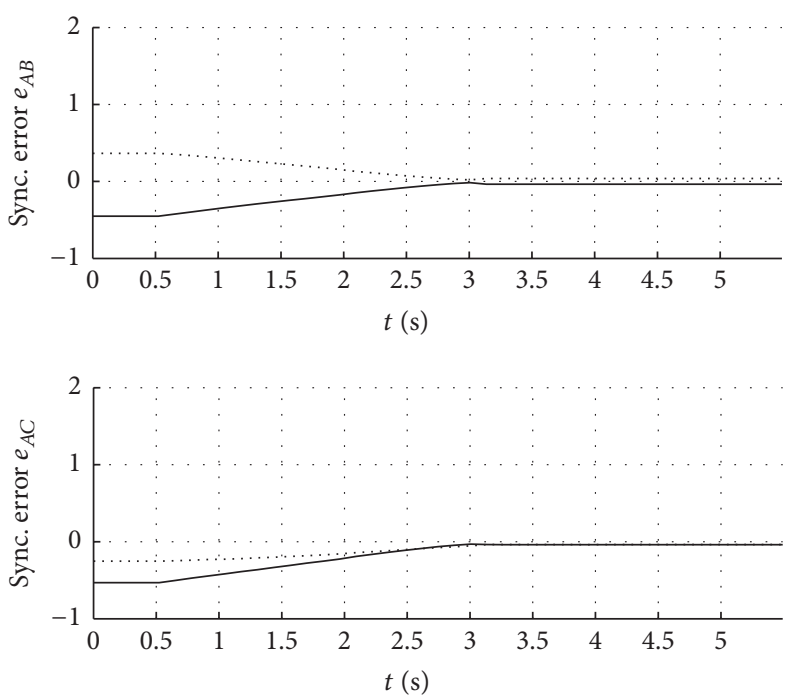

...... $x$

(a) Positions of robots

(b) Synchronization error

FiguRE 7: Experimental results for three robots with controller (40).

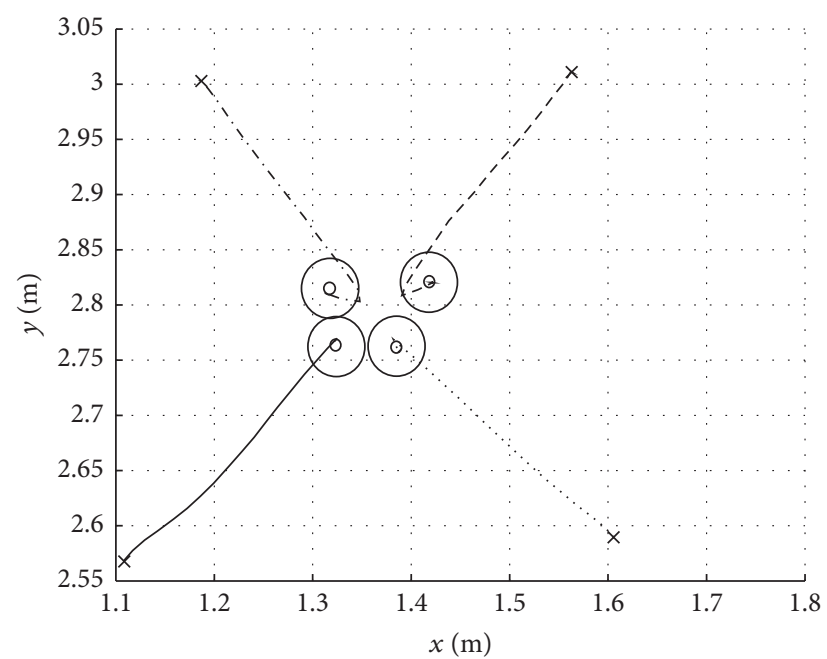

$-A$

$$
---C
$$$$
\cdots D
$$

(a) Positions of robots
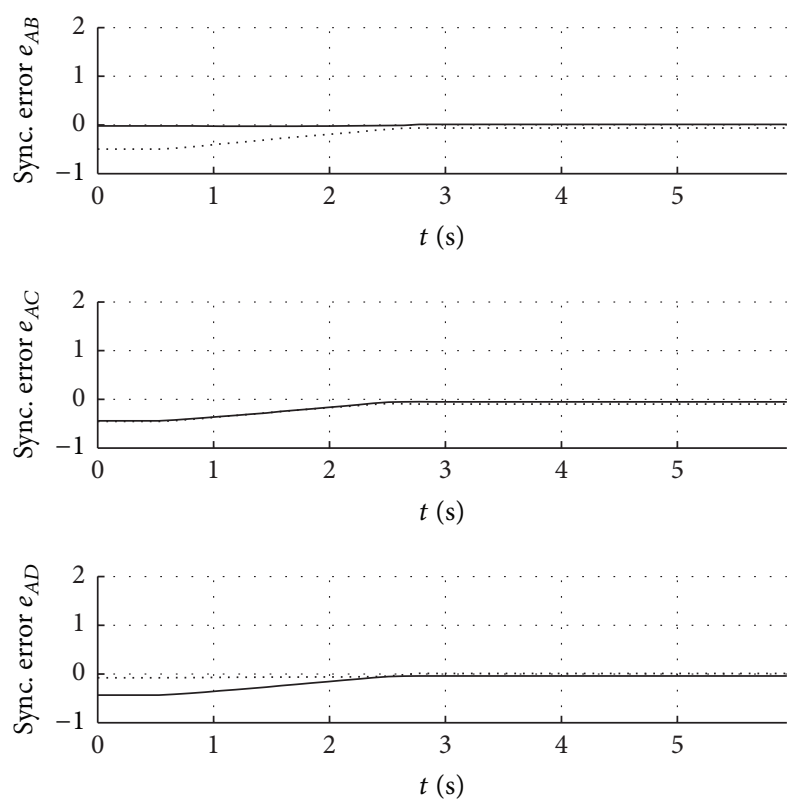

…. $x$

(b) Synchronization error

FIGURE 8: Experimental results for four robots with controller (40).

From the experimental results in Figure 8, we confirmed that consensus is achieved. Figure $8(\mathrm{a})$ shows the trajectories of the four robots that converge to one point. Figure 8(b) shows that the robots converge to consensus.

Figure 7 shows an experimental result for the same network topology, coupling strength, prediction gain, and initial conditions as the simulation shown in Figure 2 by using the robots. In Figures 2(b) and 7(b), the synchronization errors both have the tendency of convergence to zero and the agents finally achieve consensus. In the experiment, we considered the volume of the robot and the collision problem. The achieved synchronization error is between $\left[d_{\min }, d_{\max }\right]$ 
due to the controller (37) in Figure 7(b). In Figures 3 and 8, the similar results are obtained for four agents.

Remark 6. In real applications, time-delay in network communication may be time-varying and/or unknown. According to the experimental results in [18], Internet-induced time delays between different countries are almost constant. If the variation of time-varying delay is relatively slower comparing with the velocity of convergence of prediction error, the delay can be considered as constant. In this case, the proposed method is available for the MAS using communication networks. Even if the practical time-delay is time-varying, it is possible to add artificial delay to true up the length of delay to a constant value and adopt the proposed scheme by overestimating the maximum size of delay.

\section{Conclusions}

In this paper, we considered the consensus problem of MAS with input and output time delays. A controller with a state predictor based on anticipating synchronization was proposed for this system. The consensus conditions for the controller were given, and we discussed the average consensus. We concluded that the proposed controller and predictor could cope with longer time delays, since the number of robots increased. We provided numerical simulations to show the validity of the control scheme. Validity was further confirmed in experiments with nonholonomic mobile robots based on the theoretical stability criteria and collision avoidance mechanism. It was shown that the validity of the proposed predictor-based controller could be used in real applications to control multiple mobile robots converging to consensus. In this study, to apply the predictor-based control approach, time-delay is considered as a constant value. Since time-delay is variable in real applications, we would like to discuss this problem in the future study.

\section{Competing Interests}

The authors declare that they have no competing interests.

\section{Acknowledgments}

This research is an extended version of a conference paper [2] which was presented at the 2015 IEEE Multi-Conference on Systems and Control. This work was partially supported by the Japan Society for the Promotion of Science (JSPS) Grantin-Aid for Scientific Research (no. 26420424).

\section{References}

[1] W. Qiao and R. Sipahi, "Consensus control under communication delay in a three-robot system: design and experiments," IEEE Transactions on Control Systems Technology, vol. 24, no. 2, pp. 687-694, 2016.

[2] Y. Cao, T. Oguchi, P. B. Verhoeckx, and H. Nijmeijer, "Predictorbased consensus control of a multi-agent system with timedelays," in Proceedings of the IEEE Conference on Control and Applications (CCA '15), pp. 113-118, Sydney, Australia, September 2015.
[3] S. Ferrari, G. Foderaro, P. Zhu, and T. A. Wettergren, "Distributed optimal control of multiscale dynamical systems: a tutorial," IEEE Control Systems, vol. 36, no. 2, pp. 102-116, 2016.

[4] G. Antonelli, F. Arrichiello, and S. Chiaverini, "Flocking for multi-robot systems via the Null-space-based behavioral control," Swarm Intelligence, vol. 4, no. 1, pp. 37-56, 2010.

[5] N. Moshtagh, A. Jadbabaie, and K. Daniilidis, "Vision-based control laws for distributed flocking of nonholonomic agents," in Proceedings of the IEEE International Conference on Robotics and Automation (ICRA '06), pp. 2769-2774, IEEE, Orlando, Fla, USA, May 2006.

[6] W. Ren, "Multi-vehicle consensus with a time-varying reference state," Systems and Control Letters, vol. 56, no. 7-8, pp. 474-483, 2007.

[7] K. D. Listmann, M. V. Masalawala, and J. Adamy, "Consensus for formation control of nonholonomic mobile robots," in Proceedings of the IEEE International Conference on Robotics and Automation (ICRA '09), pp. 3886-3891, Kobe, Japan, May 2009.

[8] G.-B. Dai and Y.-C. Liu, "Leaderless and leader-following consensus for networked mobile manipulators with communication delays," in Proceedings of the IEEE Conference on Control and Applications (CCA '15), pp. 1656-1661, IEEE, Sydney, Australia, September 2015.

[9] Y. Cai, Q. Zhan, and X. Xi, "Path tracking control of a spherical mobile robot," Mechanism and Machine Theory, vol. 51, pp. 5873, 2012.

[10] Y. Lan, G. Yan, and Z. Lin, "Synthesis of distributed control of coordinated path following based on hybrid approach," Institute of Electrical and Electronics Engineers. Transactions on Automatic Control, vol. 56, no. 5, pp. 1170-1175, 2011.

[11] T. Oguchi and H. Nijmeijer, "Control of nonlinear systems with time-delay using state predictor based on synchronization," in Proceedings of the Euromech Nonlinear Dynamics Conference (ENOC '05), pp. 1150-1156, Eindhoven, The Netherlands, August 2005.

[12] R. Olfati-Saber and R. M. Murray, "Consensus problems in networks of agents with switching topology and time-delays," Institute of Electrical and Electronics Engineers. Transactions on Automatic Control, vol. 49, no. 9, pp. 1520-1533, 2004.

[13] R. Olfati-Saber, J. A. Fax, and R. M. Murray, "Consensus and cooperation in networked multi-agent systems," Proceedings of the IEEE, vol. 95, no. 1, pp. 215-233, 2007.

[14] A. Papachristodoulou, A. Jadbabaie, and U. Munz, "Effects of delay in multi-agent consensus and oscillator synchronization," IEEE Transactions on Automatic Control, vol. 55, no. 6, pp. 1471$1477,2010$.

[15] X. Liu, W. Lu, and T. Chen, "Consensus of multi-agent systems with unbounded time-varying delays," Institute of Electrical and Electronics Engineers. Transactions on Automatic Control, vol. 55, no. 10, pp. 2396-2401, 2010.

[16] K. H. Movric and F. L. Lewis, "Cooperative optimal control for multi-agent systems on directed graph topologies," IEEE Transactions on Automatic Control, vol. 59, no. 3, pp. 769-774, 2014.

[17] K. Kojima, T. Oguchi, A. Alvarez-Aguirre, and H. Nijmeijer, "Predictor-based tracking control of a mobile robot with timedelays," in Proceedings of the 8th IFAC Symposium on Nonlinear Control Systems (NOLCOS '10), pp. 167-172, Bologna, Italy, September 2010

[18] A. Alvarez-Aguirre, N. Van De Wouw, T. Oguchi, and H. Nijmeijer, "Predictor-based remote tracking control of a mobile 
robot," IEEE Transactions on Control Systems Technology, vol. 22, no. 6, pp. 2087-2102, 2014.

[19] K. Sakurama and K. Nakano, "Average-consensus problem for networked multi-agent systems with heterogeneous timedelays," IFAC Proceedings Volumes, vol. 44, no. 1, pp. 2368-2375, 2011.

[20] R. Wei and W. B. Randal, Distributed Consensus in Multi-Vehicle Cooperative Control, Springer, London, UK, 2008.

[21] F. Mondada, M. Bonani, X. Raemy et al., "The e-puck, a robot designed for education in engineering," in Proceedings of the 9th Conference on Autonomous Robot Systems and Competitions, pp. 59-65, Castelo Branco, Portugal, May 2009.

[22] E. Rimon and D. E. Koditschek, "Exact robot navigation using artificial potential functions," IEEE Transactions on Robotics and Automation, vol. 8, no. 5, pp. 501-518, 1992. 


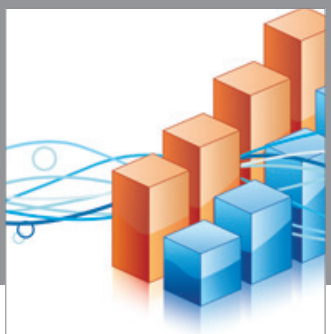

Advances in

Operations Research

vatem alat4

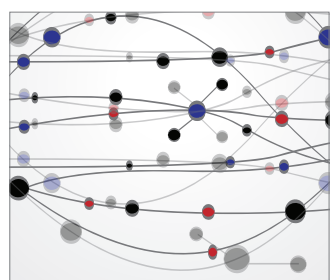

\section{The Scientific} World Journal
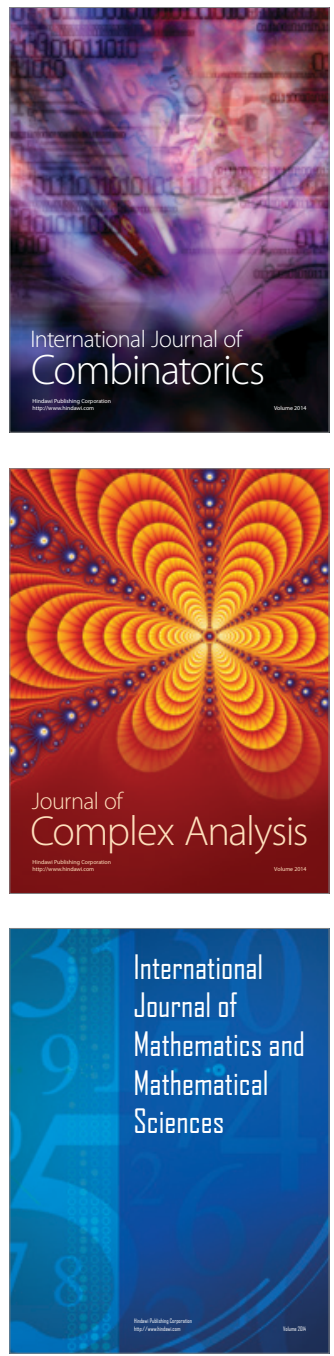
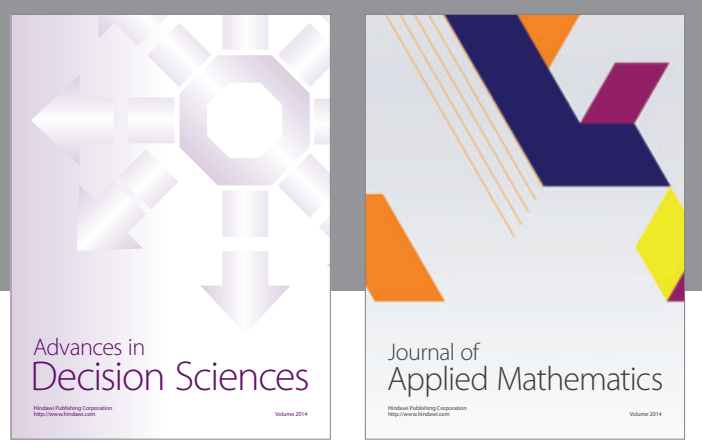

Algebra

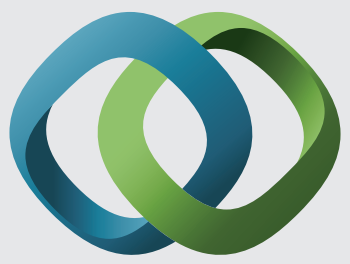

\section{Hindawi}

Submit your manuscripts at

https://www.hindawi.com
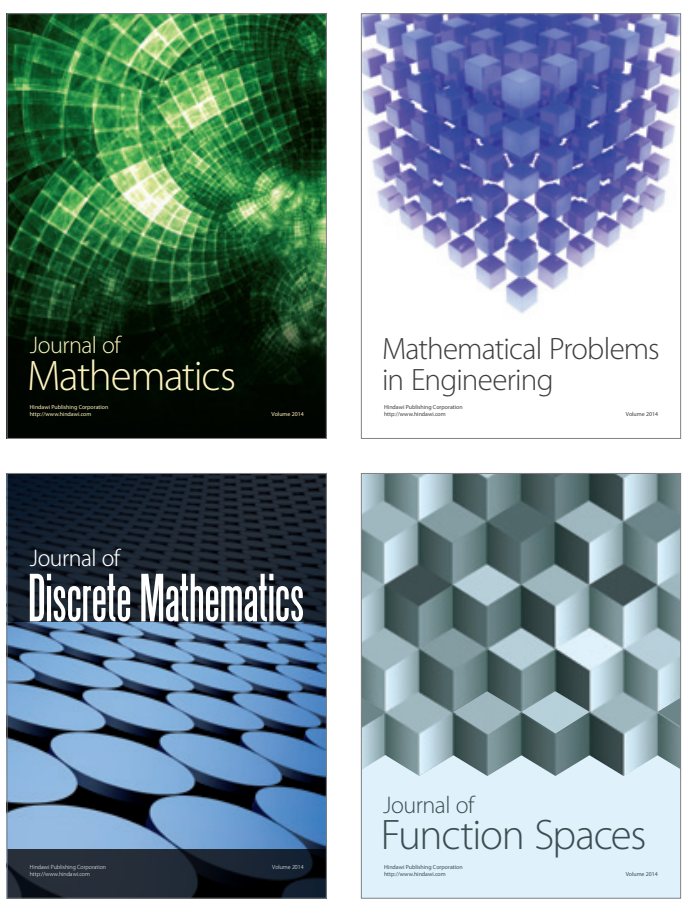

Mathematical Problems in Engineering
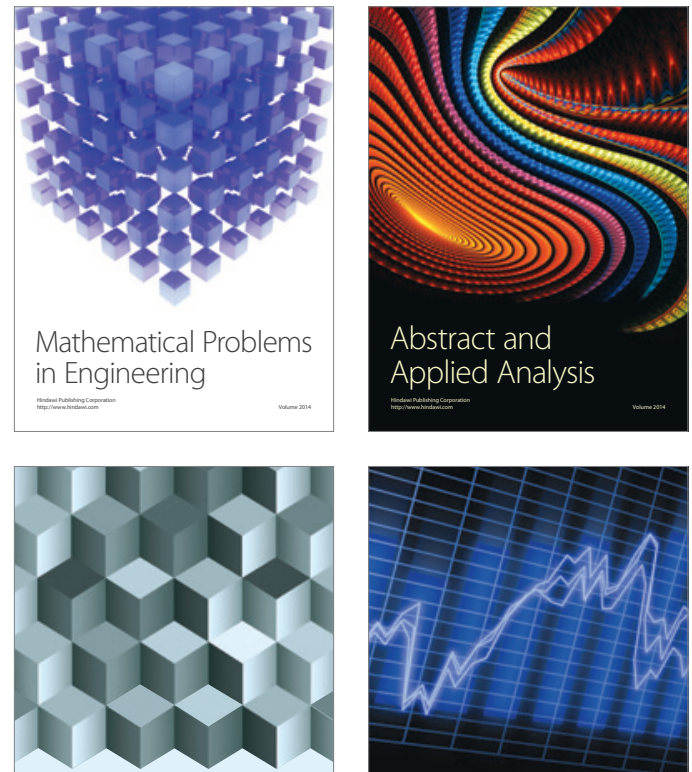

Journal of

Function Spaces

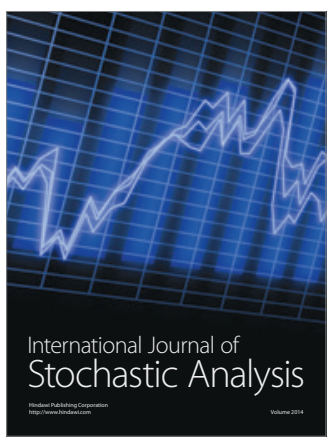

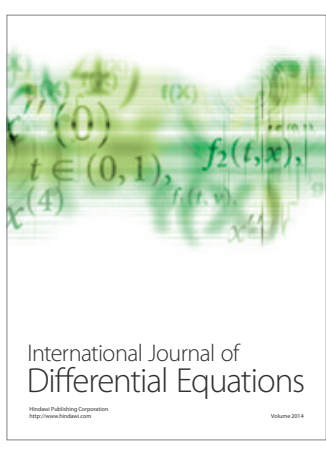
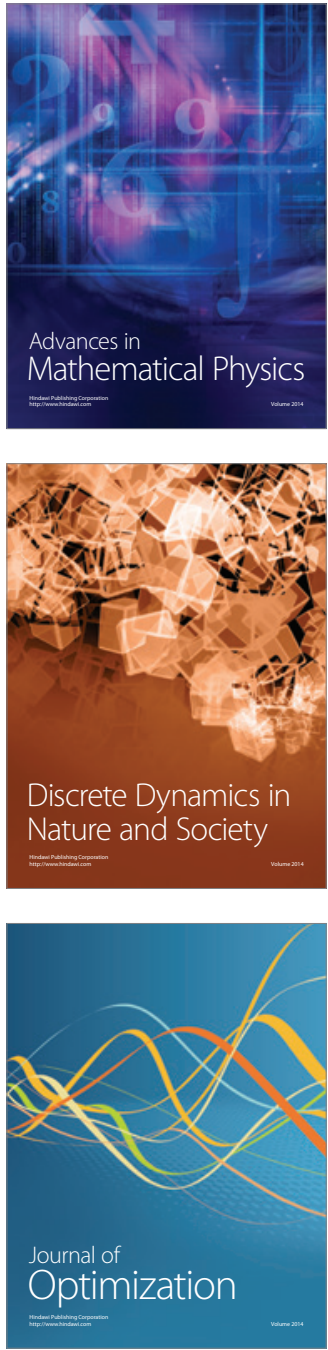